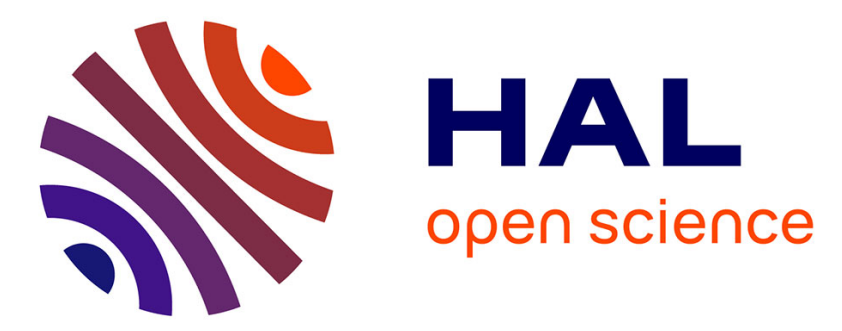

\title{
Gauge origin independent calculations of molecular magnetisabilities in relativistic four-component theory
}

Miroslav Iliaš, Hans Jørgen Aa. Jensen, Radovan Bast, Trond Saue

\section{To cite this version:}

Miroslav Iliaš, Hans Jørgen Aa. Jensen, Radovan Bast, Trond Saue. Gauge origin independent calculations of molecular magnetisabilities in relativistic four-component theory. Molecular Physics, 2013, 111 (9-11), pp.1373-1381. 10.1080/00268976.2013.798436 . hal-00880563

\section{HAL Id: hal-00880563 https://hal.science/hal-00880563}

Submitted on 28 Jan 2020

HAL is a multi-disciplinary open access archive for the deposit and dissemination of scientific research documents, whether they are published or not. The documents may come from teaching and research institutions in France or abroad, or from public or private research centers.
L'archive ouverte pluridisciplinaire HAL, est destinée au dépôt et à la diffusion de documents scientifiques de niveau recherche, publiés ou non, émanant des établissements d'enseignement et de recherche français ou étrangers, des laboratoires publics ou privés. 


\title{
MANUSCRIPT
}

\section{Gauge origin independent calculations of molecular magnetizabilities in relativistic four-component theory}

\author{
Miroslav Iliaš ${ }^{a}$, Hans Jørgen Aa. Jensen ${ }^{b}$, Radovan Bast ${ }^{c}$, and Trond Saue ${ }^{c *}$ \\ ${ }^{a}$ Department of Chemistry, Faculty of Natural Sciences, Matej Bel University, \\ Tajovského 40, SK-974 00 Banská Bystrica, Slovakia; ${ }^{b}$ Department of Physics, Chemistry \\ and Pharmacy, University of Southern Denmark, Campusvej 55, DK-5230 Odense M, \\ Denmark; ${ }^{c}$ Laboratoire de Chimie et Physique Quantique (UMR 5626), \\ CNRS/Université de Toulouse 3 (Paul Sabatier), \\ 118 route de Narbonne, F-31062 Toulouse, France \\ (Received 00 Month 200x; final version received 00 Month 200x)
}

\begin{abstract}
The use of magnetic-field dependent London atomic orbitals, also called gauge including atomic orbitals, is known to be an efficient choice for accurate non-relativistic calculations of magnetizabilities. In this work the appropriate formulas were extended and implemented in the framework of the four-component relativistic linear response method at the self-consistent field single reference level. Benefits of employing the London atomic orbitals in relativistic calculations are illustrated with Hartree-Fock wave functions on the $\mathrm{XF}_{3}(\mathrm{X}=\mathrm{N}, \mathrm{P}, \mathrm{As}, \mathrm{Sb}$, $\mathrm{Bi}$ ) series of molecules. Significantly better convergence of magnetizabilities with respect to the basis set size is observed compared to calculations employing a common gauge origin. In fact, it is mandatory to use London atomic orbitals unless you want to use ridiculously large basis sets. Relativistic effects on magnetizabilities are found to be quite small $(<5 \%)$ for this particular set of molecules, but should be investigated on a larger set of molecules. We emphasize the breakdown of the connection between the paramagnetic contribution to magnetizabilities and rotational $g$ tensors in the relativistic domain and discuss its origin. Finally, we visualize the magnetizability density which shows markedly atomic features evocative of Pascal's rules.
\end{abstract}

Keywords: molecular properties; magnetic properties; molecular magnetizabilities; relativistic effects; London atomic orbitals; property surfaces

\section{Introduction}

Presently the preferred way to ensure that calculated magnetic properties of molecules, such as magnetizabilities, are independent of the gauge origin of the external magnetic field, is through the use of the physically motivated magneticfield dependent London atomic orbitals (LAOs) [1 7], also called gauge including atomic orbitals (GIAOs) [8]. LAO-based approaches for the calculation of magnetizabilities have accordingly been reported with non-relativistic Hamiltonians at the Hartree-Fock (HF) [9], multiconfiguration self-consistent field (MCSCF) [10], density functional theory (DFT) [11 14, and coupled cluster $\operatorname{CCSD}(\mathrm{T})$ 15] levels. Schwerdtfeger and co-workers included relativistic effects with relativistic pseudopotentials, but noted a breakdown of the pseudopotential approximation in LAO-based calculations of magnetizabilities of $\mathrm{Au}, \mathrm{AuF}$, and $\mathrm{Sn}_{n}$ clusters [16]. Proper correction terms were derived by van Wüllen [17] and successfully applied in a joint publication with the previous authors [18. Yoshizawa and Hada [19 21]

\footnotetext{
*Corresponding author. Email: trond.saue@irsamc.ups-tlse.fr
} 
have reported HF and MP2 calculations of molecular magnetizabilities based on the second-order Douglas-Kroll-Hess Hamiltonian, using a common gauge origin (CGO) combined with extended basis sets [22].

In the present work we present the theory for an LAO-based calculation of magnetizabilities at the 4-component relativistic HF level, our implementation of the theory, and sample applications. This work is a natural extension of our previous development of a method for gauge origin independent 4-component relativistic calculations of NMR shielding constants [23, 24]. As a first application we have chosen to study the magnetizability of the trifluorides of the group 15 elements. Our choice was motivated by the study of Ruud and Helgaker [25] in which they demonstrated the crucial role of LAOs for the proper convergence of the magnetizability of $\mathrm{PF}_{3}$. The paper is organized as follows: In Section 2 the theory for 4-component relativistic HF calculations of magnetizabilities is derived. Computational details are given in Section 3, whereas results are presented and discussed in Section 4. Finally we conclude and provide perspectives in Section 5 .

\section{Theory}

The static $3 \times 3$ magnetizability tensor may be defined as the second derivative of the molecular energy with respect to the components of the magnetic field:

$$
\zeta_{\alpha \beta}=-\left.\frac{\mathrm{d}^{2} E}{\mathrm{~d} B_{\alpha} \mathrm{d} B_{\beta}}\right|_{\mathbf{B}=\mathbf{0}}
$$

In the present work we employ SI-based atomic units [26] in which the magnetizability is given in units of $e^{2} a_{0}^{2} / m_{e}=7.89104 \cdot 10^{-29} \mathrm{JT}^{-2}=4.752042 \cdot 10^{-6} \mathrm{erg} G^{-2}$ $\mathrm{mol}^{-1}$. We will also make extensive use of the Einstein convention of summation over repeated indices.

Our starting point is the 4-component Dirac-Coulomb (DC) Hamiltonian extended by a uniform external magnetic field $\mathbf{B}$ which is represented through the vector potential

$$
\mathbf{A}_{G}(\mathbf{r})=\frac{1}{2} \mathbf{B} \times \mathbf{r}_{G} ; \quad \mathbf{r}_{G}=\mathbf{r}-\mathbf{R}_{G}
$$

where the subscript $G$ refers to the arbitrarily chosen gauge origin. The subsequent development, restricted to closed-shell HF theory, follows closely that of the seminal paper by Helgaker and Jørgensen [7] (we note that the implementation can be straightforwardly extended to DFT). We employ an exponential parametrization of a single reference Slater determinant in terms of the orbital rotation operator $\hat{\kappa}$

$$
|0(\mathbf{B})\rangle=\exp [-\hat{\kappa}]|0(\mathbf{0})\rangle ; \quad \hat{\kappa}=\kappa_{a i} a^{\dagger} i-\kappa_{a i}^{*} i^{\dagger} a .
$$

Here and in the following we will use indices $i, j, \ldots$ and $a, b, \ldots$ for occupied and virtual orbitals, respectively, and reserve $p, q, \ldots$ for general orbitals. The molecular orbitals will be expanded in the magnetic-field dependent LAOs

$$
\omega_{\mu}(\mathbf{r})=\exp \left[-\mathrm{i} e \mathbf{A}_{G}\left(\mathbf{R}_{\mu}\right) \cdot \mathbf{r}\right] \chi_{\mu}(\mathbf{r})
$$

with $\mathbf{R}_{\mu}$ representing the center of the basis function $\chi_{\mu}$. In our case each LAO is formally a 4-component vector function (a spinor), however, only one of the four components is non-zero. An immediate advantage of using such an effectively scalar 
basis set expansion is that it allows for a straightforward use of non-relativistic integral codes. In the LAO basis the integrals of the one-electron part of the DC Hamiltonian are given by

$$
h_{\mu \nu}=\left\langle\chi_{\mu}\left|\exp \left[\frac{\mathrm{i}}{2} \mathbf{B} \cdot\left(\mathbf{R}_{\mu \nu} \times \mathbf{r}\right)\right]\left(\hat{h}_{\mathrm{D}}-\mathbf{B} \cdot \hat{\mathbf{m}}_{\nu}\right)\right| \chi_{\nu}\right\rangle=h_{\mathrm{D} ; \mu \nu}-\mathbf{B} \cdot \mathbf{m}_{\nu ; \mu \nu},
$$

where appear integrals over the Dirac Hamiltonian $\hat{h}_{\mathrm{D}}$ in the molecular field, that is, the electrostatic field of fixed nuclei, as well as over the magnetic dipole operator $\hat{\mathbf{m}}_{\nu}$ with respect to center $\mathbf{R}_{\nu}$ of the basis function $\chi_{\nu}$

$$
\hat{\mathbf{m}}_{\nu}=-\frac{1}{2}\left(\mathbf{r}_{\nu} \times c \boldsymbol{\alpha}\right) .
$$

One may note that the imaginary phase factor in the above integral vanishes for one-center integrals due to the presence of the vector $\mathbf{R}_{\mu \nu}=\mathbf{R}_{\mu}-\mathbf{R}_{\nu}$. The corresponding integrals over the two-electron Coulomb term are

$$
g_{\mu \nu, \rho \sigma}=\left\langle\chi_{\mu} \chi_{\rho}\left|\exp \left[\frac{\mathrm{i}}{2} \mathbf{B} \cdot\left(\mathbf{R}_{\mu \nu} \times \mathbf{r}_{1}+\mathbf{R}_{\rho \sigma} \times \mathbf{r}_{2}\right)\right] \frac{1}{r_{12}}\right| \chi_{\nu} \chi_{\sigma}\right\rangle .
$$

Simplification of the ensuing formalism is obtained by first defining a set of unmodified molecular orbitals (UMOs) in terms of the zero-field molecular coefficients [27, 28]

$$
\psi_{q}(\mathbf{r}, \mathbf{B})=\omega_{\mu}(\mathbf{r}, \mathbf{B}) c_{\mu q}(\mathbf{0}) .
$$

The electronic Hamiltonian in second-quantized form will, however, be constructed from a set of orthonormalized molecular orbitals (OMOs) (denoted by tilde)

$$
\tilde{\psi}_{p}(\mathbf{r}, \mathbf{B})=\psi_{q}(\mathbf{r}, \mathbf{B}) T_{q p}(\mathbf{B}) ; \quad\left\langle\tilde{\psi}_{p} \mid \tilde{\psi}_{q}\right\rangle=T_{r p}^{*}\left\langle\psi_{r} \mid \psi_{s}\right\rangle T_{s q}=\delta_{p q},
$$

which conserves the algebra of creation and annihilation operators at any field strength. Orthonormality of the OMOs at any field strength is assured by the connection matrix $T$. Throughout this work we will employ the natural connection [10, 29, 30, which provides maximum similarity in a least-squares sense between the OMOs and the unperturbed UMOs. In the OMO basis the electronic Hamiltonian may be separated into two parts

$$
\tilde{H}=\tilde{H}_{0}-\mathbf{B} \cdot \tilde{\mathbf{m}}_{p q} p^{\dagger} q ; \quad \tilde{H}_{0}=\tilde{h}_{\mathrm{D} ; p q} p^{\dagger} q+\frac{1}{2} \tilde{g}_{p q, r s} p^{\dagger} r^{\dagger} s q
$$

and it should be noted that this separation is different from the common gauge origin (CGO) case where would appear integrals of the magnetic dipole operator $\hat{\mathbf{m}}_{G}$ with respect to the arbitrary gauge origin and not the individual basis function centers as here.

The HF energy now depends explicitly on the magnetic field through the LAOs in addition to the magnetic interaction term of the Hamiltonian, Eq. (10), and implicitly through the orbital rotation parameters and the connection matrix. At any field strength we impose that the HF energy is optimized with respect to non-redundant orbital rotation parameters $\left\{\kappa_{p q}\right\}$. The components of the magnetizability tensor, Eq. (1), are accordingly given by

$$
\zeta_{\alpha \beta}=\zeta_{\alpha \beta}^{\text {linres }}+\zeta_{\alpha \beta}^{\text {expval }}
$$


The linear response part is given by

$$
\zeta_{\alpha \beta}^{\text {linres }}=-\left.\left\langle 0\left|\left[\frac{\mathrm{d} \boldsymbol{\kappa}}{\mathrm{d} B_{\beta}},\left(\frac{\mathrm{d} \tilde{H}_{0}}{\mathrm{~d} B_{\alpha}}-\tilde{m}_{\alpha}\right)\right]\right| 0\right\rangle\right|_{\mathbf{B}=\mathbf{0}}=-\left(\kappa_{a i}^{\beta ; *} \tilde{F}_{a i}^{\alpha}+\tilde{F}_{i a}^{\alpha} \kappa_{a i}^{\beta}\right)
$$

where appear magnetic derivatives of the OMO Fock matrix

$$
\tilde{F}_{p q}^{\alpha}=\left.\frac{\mathrm{d} \tilde{F}_{p q}}{\mathrm{~d} B_{\alpha}}\right|_{\mathbf{B}=\mathbf{0}}=-m_{\alpha ; p q}+\tilde{h}_{\mathrm{D} ; p q}^{\alpha}+\tilde{\mathcal{L}}_{p q, j j}^{\alpha} ; \quad \mathcal{L}_{p q, r s}=(p q \mid r s)-(p s \mid r q) .
$$

The non-redundant first-order amplitudes $\kappa^{\beta}$ are found by solving a response equation involving the generalized property gradient $\tilde{F}^{\beta}$ as described in Ref. 23. The expectation value part is given by

$$
\begin{aligned}
\zeta_{\alpha \beta}^{\text {expval }} & =-\left.\left\langle 0\left|\frac{\mathrm{d}^{2} \tilde{H}_{0}}{\mathrm{~d} B_{\alpha} \mathrm{d} B_{\beta}}-\frac{\mathrm{d} \tilde{m}_{\alpha}}{\mathrm{d} B_{\beta}}-\frac{\mathrm{d} \tilde{m}_{\beta}}{\mathrm{d} B_{\alpha}}\right| 0\right\rangle\right|_{\mathbf{B}=\mathbf{0}} \\
& =-\left(\tilde{h}_{\mathrm{D} ; i i}^{\alpha \beta}+\frac{1}{2} \tilde{\mathcal{L}}_{i i, j j}^{\alpha \beta}\right)+P_{\alpha \beta} \tilde{m}_{\alpha, i i}^{\beta},
\end{aligned}
$$

where $P_{\alpha \beta}$ symmetrizes the expression with respect to components $\alpha$ and $\beta$ of the magnetic field. The first- and second-order magnetic derivatives appearing in the above expressions can be expressed compactly using the brace notation of Helgaker and Jørgensen [7] for the one-index transformation of one- and two-electron matrix elements

$$
\begin{aligned}
\left\{T^{\alpha}, h\right\}_{p q} & =T_{p^{\prime} p}^{\alpha *} h_{p^{\prime} q}+h_{p q^{\prime}} T_{q^{\prime} q}^{\alpha} \\
\left\{T^{\alpha}, \mathcal{L}\right\}_{p q, r s} & =T_{p^{\prime} p}^{\alpha *} \mathcal{L}_{p^{\prime} q, r s}+\mathcal{L}_{p q^{\prime}, r s} T_{q^{\prime} q}^{\alpha}+T_{r^{\prime} r}^{\alpha *} \mathcal{L}_{p q, r^{\prime} s}+\mathcal{L}_{p q, r s^{\prime}} T_{s^{\prime} s}^{\alpha}
\end{aligned}
$$

For one-electron operators in OMO basis the first and second magnetic derivatives are then given $a s^{1}$

$$
\begin{aligned}
\tilde{h}_{\mathrm{D} ; m n}^{\alpha} & =h_{\mathrm{D} ; m n}^{\alpha}+\left\{T^{\alpha}, h_{\mathrm{D}}\right\}_{m n} \\
\tilde{h}_{\mathrm{D} ; m n}^{\alpha \beta} & =h_{\mathrm{D} ; m n}^{\alpha \beta}+P_{\alpha \beta}\left\{T^{\alpha}, h_{\mathrm{D}}^{\beta}\right\}_{m n}+\left\{T^{\alpha \beta}, h_{\mathrm{D}}\right\}_{m n} \\
& +\frac{1}{2} P_{\alpha \beta}\left(\left\{T^{\alpha},\left\{T^{\beta}, h_{\mathrm{D}}\right\}\right\}_{m n}-\left\{T^{\beta} T^{\alpha}, h_{\mathrm{D}}\right\}_{m n}\right) .
\end{aligned}
$$

The corresponding expressions for two-electron operators are equivalent, but involve four indices and the brace notation of Eq. (16).

The final expressions, Eqs. (12) and (14), for components of the magnetizability tensor are quite similar to non-relativistic theory [7, 9, 10, but involve some new integrals such as the first- and second derivatives of the Dirac Hamiltonian in LAO-basis

$$
\begin{aligned}
& h_{\mathrm{D} ; \mu \nu}^{\alpha}=\frac{\mathrm{i}}{2}\left\langle\chi_{\mu}\left|\left(\mathbf{R}_{\mu \nu} \times \mathbf{r}\right)_{\alpha} \hat{h}_{\mathrm{D}}\right| \chi_{\nu}\right\rangle, \\
& h_{\mathrm{D} ; \mu \nu}^{\alpha \beta}=-\frac{1}{4}\left\langle\chi_{\mu}\left|\left(\mathbf{R}_{\mu \nu} \times \mathbf{r}\right)_{\alpha}\left(\mathbf{R}_{\mu \nu} \times \mathbf{r}\right)_{\beta} \hat{h}_{\mathrm{D}}\right| \chi_{\nu}\right\rangle,
\end{aligned}
$$

\footnotetext{
${ }^{1}$ These expressions may be compared to Eqs. (32) and (33) of Ref. 10 it will be seen that for the second magnetic derivative there is a sign difference in the second term which we believe is correct for our expression.
} 
which has been implemented in the integral driver HERMIT [31 in a development version of the DIRAC12 code for relativistic molecular calculations [32. An important difference, though, is that the relativistic expression does not contain an explicit diamagnetic term. In a CGO formalism the diamagnetic contribution can be extracted from the part of the linear response function involving rotations between occupied positive-energy and virtual negative-energy orbitals [33, 34]. In an LAO-based formalism the situation is somewhat more complicated since reorthonormalization of UMOs, Eq. (8), involve both positive- and negative-energy orbitals. The separation of para- and diamagnetic contributions in a LAO framework will not be pursued in the present work.

\section{Computational details}

Using a development version of the DIRAC12 code [32] we have calculated HF magnetizabilities of the series of molecules $\mathrm{XF}_{3}(\mathrm{X}=\mathrm{N}, \mathrm{P}, \mathrm{As}, \mathrm{Sb}, \mathrm{Bi})$ in different basis sets of increasing size. We have employed the experimental structures, as given in Table 1, all corresponding to trigonal pyramidal structures. Most calculations were based on the 4-component DC Hamiltonian, but in order to investigate separately scalar and spin-orbit effects on magnetizabilities we have also performed spin-orbit free and non-relativistic calculations based on the modified Dirac [35, 36] and the Lévy-Leblond [37] Hamiltonians, respectively. In addition, for non-relativistic reference values we have performed calculations with the Dalton [38] code. In all calculations we have chosen a Gaussian model for the nuclear charge distributions [39].

For the lighter atoms (F, N, P and As) we have employed sequences of precc-pVXZ basis sets of Dunning and coworkers [40 43], where pre may indicate single $(a u g)$ and double (d-aug) augmentation. For the heavier atoms $\mathrm{Sb}$ and $\mathrm{Bi}$ we employed the corresponding basis sets by Dyall [44, 45], denoted dyall.vnz ( $n=2,3,4$, dyall. $a \mathrm{v} n \mathrm{z}$ with augmentation) in the DIRAC basis set library. In this work we will specify basis sets with the notation pre-XZ which is to be understood as an alias for both the Dunning basis sets (F, N, P and As) and the Dyall basis sets ( $\mathrm{Sb}$ and $\mathrm{Bi}$ ). All basis sets have been employed in uncontracted form. In the DIRAC code the small component basis sets were generated by restricted kinetic balance (RKB) in the SCF step and extended according to the simple magnetic balance (sMB) scheme in the magnetizability calculations [24]. The $(S S \mid S S)$ class of twoelectron integrals was neglected throughout the four-component DC relativistic calculations. For the LAO calculations we used the natural connection [29] in the reorthonormalization terms. In the CGO calculations the gauge origin was placed at the center of mass of the molecule.

\section{Results and discussion}

In Table 2 we report our calculated magnetizabilities for the selected molecules $\mathrm{XF}_{3}(\mathrm{X}=\mathrm{N}, \mathrm{P}, \mathrm{As}, \mathrm{Sb}, \mathrm{Bi})$. In addition to parallel $\left(\zeta_{\|}\right)$and perpendicular $\left(\zeta_{\perp}\right)$ components of the magnetizability with respect to the $C_{3}$ axis, we also report the isotropic and anisotropic components defined as:

$$
\begin{aligned}
\zeta_{\text {iso }} & =\frac{1}{3}\left(\zeta_{\|}+2 \zeta_{\perp}\right) \\
\zeta_{\text {ani }} & =\zeta_{\perp}-\zeta_{\|}
\end{aligned}
$$


In Figure 1 we illustrate the convergence of $\zeta_{\text {iso }}$ of $\mathrm{BiF}_{3}$ in the basis sets $\mathrm{XZ}$ and aug-XZ. As was already shown by Ruud and Helgaker 25 for the $\mathrm{PF}_{3}$ molecule, the use of LAOs is crucial for obtaining fast convergence of magnetizabilities with respect to the basis set. Taking the $\mathrm{LAO}$ result for $\zeta_{\text {iso }}$ of $\mathrm{BiF}_{3}$ in aug-QZ basis as reference, the CGO result in the DZ basis is in error by $152 \%$, whereas the corresponding LAO number is within $1 \%$ of the reference value. Augmenting the basis with diffuse functions reduces the CGO error to $42 \%$ (aug-DZ), but even in the aug-QZ basis the CGO error with respect to the reference value is more than $6 \%$. The effect of augmentation of the basis was studied in more detail for $\mathrm{NF}_{3}$. As seen from Table 2, the calculated CGO value of $\zeta_{\text {iso }}$ is $-11.47 e^{2} a_{0}^{2} / m_{e}$ and -6.46 $e^{2} a_{0}^{2} / m_{e}$ in the DZ and aug-DZ bases, respectively. Simply adding the diffuse $d$ functions of fluorine reduces the $\mathrm{DZ}$ value to $-6.10 e^{2} a_{0}^{2} / m_{e}$, whereas the effect of adding the other diffuse functions is quite small. However, for consistency we have chosen to employ the same augmentation level for the basis sets of all atoms in this study. The LAO results are in comparison hardly sensitive to augmentation. For all molecules the isotropic magnetizability $\zeta_{\text {iso }}$ is converged to within $1 \%$ using LAOs in conjunction with the aug-TZ basis set. In passing we note that adding the $(S S \mid S S)$ class of two-electron integrals reduces both the parallel and perpendicular magnetizabilities of $\mathrm{BiF}_{3}$, obtained with LAOs in aug-QZ basis, by $0.0025 e^{2} a_{0}^{2} / m_{e}$, which is outside the precision with which our results are reported in Table 2 , thus justifying the neglect of this integral class.

Table 2 shows that relativistic effects on magnetizabilities are rather small for the selected set of molecules; for $\mathrm{BiF}_{3}$ relativity increases the isotropic magnetizability by about $5 \%$. Under the assumption of additivity of atomic susceptibilities in diamagnetic molecules, as proposed by Pascal [46 48], and taking his value of $-1.4 e^{2} a_{0}^{2} / m_{e}$ for the atomic susceptibility of fluorine [49], we find that that the atomic susceptibility increases as $-2.3 \cdot Z^{0.38} e^{2} a_{0}^{2} / m_{e}$ for the group 15 atoms. Furthermore, relativity will increase the atomic susceptibility of the bismuth atom by $7 \%$, from -9.3 to $-8.7 e^{2} a_{0}^{2} / m_{e}$. This result is in line with the rather small relativistic effects on magnetizabilities of noble gas atoms and group 14, 15, 16 and 17 hydrides reported by Yoshizawa and Hada [20, 21]. However, the same authors found relativistic effects on the order of $20 \%$ for the IF molecule. Qualitatively we can understand these observations in the following way: One would expect large effects of 4-component calculations of magnetizabilities compared to non-relativistic calculations when spin-orbit coupling is important for the valence electrons. As the fluorine atoms are very electronegative, the central atom of the $\mathrm{XF}_{3}(\mathrm{X}=\mathrm{N}, \mathrm{P}$, $\mathrm{As}, \mathrm{Sb}, \mathrm{Bi}$ ) series of molecules will have an oxidation state of +3 , that is, the valence $n p$ orbitals are formally not occupied. Indeed, projection analysis [50] of $\mathrm{BiF}_{3}$ gives a charge of +2.2 of bismuth with a valence configuration of $6 s_{1 / 2}^{1.8} 6 p_{1 / 2}^{0.5} 6 p_{3 / 2}^{0.6}$ in terms of the calculated atomic orbitals of neutral bismuth in its ground state electronic configuration. Further analysis, using the modified Dirac [35, 36] and the Lévy-Leblond [37] Hamiltonians, shows that the relativistic effect is almost completely dominated by scalar relativistic effects. On the other hand, for IF the valence $n p$ orbitals are formally occupied, so it is perhaps not surprising to observe large relativistic effects on the magnetizability of this molecule. Clearly relativistic effects on magnetizabilities merit further study.

Experimental values for our selected set of molecules are scarce, but have been included in Table 2 whenever available. Comparison of our HF results with experiment is meaningful since the effects of electron correlation and vibrational corrections are found to be generally small for magnetizabilities [13, 15, 51, 52, of diamagnetic molecules. For instance, a recent benchmark study by Lutnæs et al. [13] of a set of 28 small molecules shows that the contribution from electron 
correlation and zero-point vibrational corrections are typically within $2 \%$ and $1 \%$, respectively, but with outliers such as paramagnetic $\mathrm{O}_{3}$ and $\mathrm{BH} 15$ for correlation and $\mathrm{N}_{2}, \mathrm{CO}, \mathrm{O}_{3}, \mathrm{LiH}$, and $\mathrm{PN}$ for vibration. The anisotropic component of the magnetizability can be obtained from microwave spectroscopy of molecules in an external magnetic field [53. Experimental values of $\zeta_{\text {ani }}$ obtained in this manner have been reported for $\mathrm{NF}_{3}$ and $\mathrm{PF}_{3}$ by Stone et al. [54] and later refined for $\mathrm{PF}_{3}$ by Hüttner and co-workers [55]. For $\mathrm{NF}_{3}$ the agreement with our HF/LAO-results is quite good, but less so for $\mathrm{PF}_{3}$. For the latter molecule Stone et al. 54 provide an estimate of the isotropic contribution based on Pascal's rules. The discrepancy, on the order of 15\%, may in part be due to the possible breakdown of Pascal's rules for fluorine-containing molecules [52. Experimental data for bulk susceptibilities of $\mathrm{SbF}_{3}\left(\zeta_{\text {iso }}=-9.68 e^{2} a_{0}^{2} / m_{e}\right)$ and $\mathrm{BiF}_{3}\left(\zeta_{\text {iso }}=-12.8 e^{2} a_{0}^{2} / m_{e}\right)$ can be found in the compilation by Föex [56], referring to experimental work by Pascal [49] and Chowdhury [57], respectively, and agree reasonably well with our HF values.

In a non-relativistic framework the paramagnetic component of the magnetizability can be extracted from the rotational $g$ tensor [53] much in the same way as the paramagnetic component of the NMR shielding tensor can be related to the spin-rotation constant [58 60], and has accordingly been given for the $\mathrm{PF}_{3}$ molecule by Stone et al. [54. It is important, however, to stress that these connections do not hold in the relativistic domain [61 63]. This can be understood from the following argument: Component $\zeta_{\alpha \beta}$ of the magnetizability tensor can be interpreted as component $\alpha$ of the magnetic dipole moment induced to first order by component $\beta$ of an external magnetic field and has the general form

$$
\zeta_{\alpha \beta}=\frac{1}{2} \int \mathrm{d} \mathbf{r}\left(\mathbf{r}_{G} \times \mathbf{j}^{\left(B_{\beta}\right)}(\mathbf{r})\right)_{\alpha}
$$

The current density $\mathbf{j}$ corresponds to the electron charge times the density associated with the appropriate velocity operator, which is different in the relativistic and non-relativistic domains. Starting from the Legendre transformation

$$
\mathbf{p} \cdot \mathbf{v}=H(\mathbf{r}, \mathbf{p})+L(\mathbf{r}, \mathbf{v})
$$

one can extract the relativistic and non-relativistic velocity operators as

$$
\mathbf{v}^{\mathrm{R}}=\frac{\partial H^{\mathrm{R}}}{\partial \mathbf{p}}=c \boldsymbol{\alpha} ; \quad \mathbf{v}^{\mathrm{NR}}=\frac{\partial H^{\mathrm{NR}}}{\partial \mathbf{p}}=\frac{\boldsymbol{\pi}}{m} ; \quad \boldsymbol{\pi}=\mathbf{p}+e \boldsymbol{A}
$$

The relativistic form, in terms of the Dirac $\boldsymbol{\alpha}$ matrices scaled with the speed of light $c$, can be interpreted in terms of the Zitterbewegung superimposed on the mean trajectory of the electron [64. The non-relativistic form of the velocity operator naturally splits into a paramagnetic and diamagnetic part, where the former, inserted into Eq. (23), gives rise to an orbital angular momentum operator with respect to the gauge origin $G$. Setting the gauge origin to the center of mass of the molecule then provides the connection to the rotational $g$ tensor, a connection which is absent in the relativistic case.

Starting from Eq. 23) one may define a magnetizability density

$$
\zeta_{\alpha \beta}^{\mathrm{CGO}}(\mathbf{r})=\frac{1}{2}\left(\mathbf{r}_{G} \times \mathbf{j}^{\left(B_{\beta}\right)}(\mathbf{r})\right)_{\alpha} .
$$

which integrates to a specific component $\zeta_{\alpha \beta}$ of the magnetizability tensor. The first-order magnetically induced current density $\mathbf{j}^{\left(B_{\beta}\right)}$ can be constructed from the 
perturbed orbital rotation parameters $\kappa^{\beta}$ which appear in Eq. 12 . In the relativistic case the orbital rotation parameters split into two classes, $\left\{\kappa_{a i}^{++}\right\}$and $\left\{\kappa_{a i}^{-+}\right\}$, corresponding to rotations between the occupied positive-energy orbitals and virtual positive- and negative-energy orbitals, respectively. This separation provides a separation of the para- and diamagnetic contributions to the induced current density, as demonstrated in Ref. 34. In Figure 2 the total isotropic average of $\zeta_{\alpha \beta}^{\mathrm{CGO}}(\mathbf{r})$ in $\mathrm{BiF}_{3}$ is represented using an isosurface plot together with its para- and diamagnetic contributions using the above recipe. For technical reasons the plots are based on calculations using a common gauge origin and a large basis (aug-QZ). We indeed observe that the para- and diamagnetic densities have opposite signs. The diamagnetic contribution resembles a superposition of atomic contributions, evocative of Pascal's rules, and dominates the total isotropic average density in $\mathrm{BiF}_{3}$. While the density isosurface around $\mathrm{Bi}$ atom is negative, the contributions from the $\mathrm{F}$ atoms are polarized and exhibit both positive and negative isosurfaces. The densities corresponding to the parallel and one of the perpendicular contributions, $\zeta_{\|}^{\mathrm{CGO}}(\mathbf{r})$ and $\zeta_{\perp}^{\mathrm{CGO}}(\mathbf{r})$, are visualized in Figures 3 and 4 , respectively. Figure 3 representing the parallel component $\zeta_{\|}^{\mathrm{CGO}}(\mathbf{r})$ gives direct visual evidence that there is almost no contribution to the paramagnetic part from the Bi atom. The diamagnetic contribution on the other hand features a torus-shaped isosurface around the Bi atom.

\section{Conclusions and Perspectives}

We have presented the theory and an implementation of 4-component relativistic LAO-based HF calculations of molecular magnetizabilities. An obvious extension of the present work would be to the Kohn-Sham DFT framework. We have already implemented the DFT extension of the linear response term $\zeta_{\alpha \beta}^{\text {linres }}$, Eq. $\sqrt{12}$, and have applied it to the calculation of NMR shielding constants [24]. We will next add the missing DFT contributions to the expectation value term $\zeta_{\alpha \beta}^{\text {expval }}$, Eq. (14). It would also be interesting to explore static correlation effects in the framework of 4-component relativistic MCSCF [65. We furthermore foresee a study of the separation of para- and diamagnetic contributions of the magnetizability in an LAO-based relativistic framework.

As a first application we have calculated magnetizabilities of the $\mathrm{XF}_{3}(\mathrm{X}=\mathrm{N}, \mathrm{P}$, $\mathrm{As}, \mathrm{Sb}, \mathrm{Bi}$ ) molecules with LAOs. We observe, contrary to CGO-based calculations, fast basis set convergence in 4-component calculations, just as in the non-relativistic domain [9, 25]. Our code also allows visualization of the magnetizability density which for $\mathrm{BiF}_{3}$ shows markedly atomic features evocative of Pascal's rules. Since the effects of electron correlation and vibrational corrections are generally small for magnetizabilities [51], the agreement to experiment, when available, is quite reasonable. For the selected set of molecules relativistic effects are rather small (5\%), but a study by Yoshizawa and Hada [21] indicates that relativistic effects on magnetizabilities may reach $20 \%$. We therefore plan further studies of magnetizabilities of molecules containing heavy elements. Another interesting aspect to explore is the breakdown of the connection between rotational $g$ tensor and the paramagnetic contribution to the magnetizabilities in the relativistic domain. 


\section{ACKNOWLEDGMENTS}

It is a great pleasure to dedicate this paper to a dear and very much respected colleague, Trygve Helgaker, on his 60th birthday. According to Picasso, one starts to get young at the age of sixty and then it is too late. Trygve may prove him wrong. On a more serious note, the scientific impact of Trygve Helgaker is evident in this contribution: he wrote the first version of the integral package HERMIT [31] used for the integrals in this work back in the 1980's, and he has given significant contributions to eight of the central references in this work.

This work was done under Slovak - French Research and Development Cooperation (Grant No. SK-FR-00180-11). MI is grateful for the financial support from the Slovak Research and Development Agency (Grant No. APVV-0059-10). 


\section{References}

[1]F. London, J. Phys. Radium 8, 397 (1937).

[2] H. Hameka, Mol. Phys. 1 (3), 203 (1958).

[3] H.F. Hameka, Rev. Mod. Phys. 34 (1), 87 (1962).

[4]R. Ditchfield, J. Chem. Phys. 56 (11), 5688 (1972).

[5]R. Ditchfield, Mol. Phys. 27 (4), 789 (1974).

[6]K. Wolinski, J.F. Hinton and P. Pulay, J. Am. Chem. Soc. 112 (23), 8251 (1990).

[7]T. Helgaker and P. Jørgensen, J. Chem. Phys. 95, 2595 (1991).

[8] Aa.E. Hansen and T.D. Bouman, J. Chem. Phys. 82, 5035 (1985).

[9]K. Ruud, T. Helgaker, K.L. Bak, P. Jørgensen and H.J.Aa. Jensen, J. Chem. Phys. 99, 3847 (1993).

[10]K. Ruud, T. Helgaker, K.L. Bak, P. Jørgensen and J. Olsen, Chem. Phys. 195, 157 (1995).

[11]D.J.D. Wilson, C.E. Mohn and T. Helgaker, J. Chem. Theory Comput. 1 (5), 877 (2005).

[12] M. Krykunov and J. Autschbach, J. Chem. Phys. 126, 024101 (2007).

[13] O.B. Lutnæs, A.M. Teale, T. Helgaker, D.J. Tozer, K. Ruud and J. Gauss, J. Chem. Phys. 131 (14), 144104 (2009).

[14] M.P. Johansson and M. Swart, J. Chem. Theory Comput. 6, 3302 (2010).

[15]J. Gauss, K. Ruud and M. Kállay, J. Chem. Phys. 127, 074101 (2007).

[16]P. Schwerdtfeger, B. Assadollahzadeh, U. Rohrmann, R. Schaefer and J.R. Cheeseman, J. Chem. Phys. 134 (20), 204102 (2011).

[17]C. van Wüllen, J. Chem. Phys. 136 (11), 114110 (2012).

[18]P. Schwerdtfeger, C. van Wüllen and J.R. Cheeseman, J. Chem. Phys. 137 (1), 014107 (2012).

[19] T. Yoshizawa and M. Hada, J. Comp. Chem. 28, 740 (2007).

[20]T. Yoshizawa and M. Hada, Chem. Phys. Lett. 458, 223 (2008).

[21] T. Yoshizawa and M. Hada, J. Comp. Chem. 30, 2550 (2009).

[22] M. Sugimoto and H. Nakatsuji, J. Chem. Phys. 102 (1), 285 (1995).

[23]M. Iliaš, T. Saue, T. Enevoldsen and H.J.Aa. Jensen, J. Chem. Phys. 131, 124119 (2009).

[24] M. Olejniczak, R. Bast, T. Saue and M. Pecul, J. Chem. Phys. 136 (1), 014108 (2012), Erratum: ibid. 136, 239902, (2012).

[25]K. Ruud and T. Helgaker, Chem. Phys. Lett. 264, 17 (1997).

[26] D.H. Whiffen, Pure Appl. Chem 50, 75 (1978).

[27] J. Gerratt and I.M. Mills, J. Chem. Phys. 49 (4), 1719 (1968).

[28] J.A. Pople, R. Krishnan, H.B. Schlegel and J.S. Binkley, Int. J. Quantum. Chem. 16 (S13), 225 (1979).

[29] J. Olsen, K.L. Bak, K. Ruud, T. Helgaker and P. Jørgensen, Theoret. Chim. Acta 90, 421 (1995).

[30]K. Ruud, T. Helgaker, J. Olsen, P. Jørgensen and K.L. Bak, Chem. Phys. Lett. 235, 47 (1995).

[31]HERMIT, a molecular integral program, written by T. Helgaker, P.R. Taylor, and K. Ruud.

[32] DIRAC, a relativistic ab initio electronic structure program, Release DIRAC12 (2012), written by H. J. Aa. Jensen, R. Bast, T. Saue, and L. Visscher, with contributions from V. Bakken, K. G. Dyall, S. Dubillard, U. Ekström, E. Eliav, T. Enevoldsen, T. Fleig, O. Fossgaard, A. S. P. Gomes, T. Helgaker, J. K. Lærdahl, Y. S. Lee, J. Henriksson, M. Iliaš, Ch. R. Jacob, S. Knecht, S. Komorovský, O. Kullie, C. V. Larsen, H. S. Nataraj, P. Norman, G. Olejniczak, J. Olsen, Y. C. Park, J. K. Pedersen, M. Pernpointner, K. Ruud, P. Sałek, B. Schimmelpfennig, J. Sikkema, A. J. Thorvaldsen, J. Thyssen, J. van Stralen, S. Villaume, O. Visser, T. Winther, and S. Yamamoto (see http://www.diracprogram.org).

[33] G. Aucar, T. Saue, H.J.Aa.. Jensen and L. Visscher, J. Chem. Phys. 110, 6208 (1999).

[34]R. Bast, J. Jusélius and T. Saue, Chem. Phys. 356, 187 (2009).

[35]K.G. Dyall, J. Chem. Phys. 100, 2118 (1994).

[36] L. Visscher and T. Saue, J. Chem. Phys. 113, 3996 (2000).

[37] J.M. Lévy-Leblond, Commun. Math. Phys. 6, 286 (1967).

[38]DALTON, a molecular electronic structure program, Release Dalton2011 (2011), see http:// daltonprogram.org/

[39]L. Visscher and K.G. Dyall, At. Data Nucl. Data Tables 67, 207 (1997), see also http://www.few.vu. nl/ visscher/FiniteNuclei/FiniteNuclei.htm

[40]T.H. Dunning jr., J. Chem. Phys. 90, 1007 (1989).

[41]D.E. Woon and T.H.D. jr., J. Chem. Phys. 98, 1358 (1993).

[42] A.K. Wilson, D.E. Woon and K.A. Peterson, J. Chem. Phys. 110, 7667 (1999).

[43]R.A. Kendall, J. Thom H. Dunning and R.J. Harrison, J. Chem. Phys. 96 (9), 6796 (1992).

[44] K.G. Dyall, Theoret. Chem. Acc. 115, 441 (2006), The basis sets are available from the DIRAC web site http://diracprogram.org/

[45]K.G. Dyall, Theoret. Chem. Acc. 99, 366 (1998).

[46]P. Pascal, Ann. Chim. Phys. 19, 5 (1910).

[47]P. Pascal, Ann. Chim. Phys. 25, 289 (1912).

[48]P. Pascal, Ann. Chim. Phys. 29, 218 (1913).

[49] P. Pascal, C. r. hebd. séances Acad. sci. 218, 57 (1944).

[50]S. Dubillard, J.B. Rota, T. Saue and K. Fægri, J. Chem. Phys. 124, 154307 (2007).

[51]K. Ruud, T. Helgaker and P.J. rgensen, J. Chem. Phys. 107 (24), 10599 (1997).

[52]K. Ruud, P.O. Astrand and P.R. Taylor, J. Phys. Chem. A 105, 9926 (2001).

[53]W. Hüttner and W.H. Flygare, J. Chem. Phys. 47 (10), 4137 (1967).

[54] R.G. Stone, J.M. Pochan and W.H. Flygare, Inorg. Chem. 8, 2647 (1969).

[55] W. Hüttner, P. Nowicki and M. Gamperling, Chem. Phys. Lett. 288, 553 (1998).

[56] G. Föex, Constantes Sélectionnées. Diamagnétisme et Paramagnétisme., Vol. 7 (Masson and Co., Paris, 1957).

[57]A A Chowdhury, Current Sci., India 8 (12), 550 (1939).

[58] N.F. Ramsey, Phys. Rev. 78, 699 (1950).

[59] W.H. Flygare, J. Chem. Phys. 41 (3), 793 (1964). 
[60]T.D. Gierke and W.H. Flygare, J. Am. Chem. Soc. 94 (21), 7277 (1972).

[61]L. Visscher, T. Enevoldsen, T. Saue, H.J.Aa. Jensen and J. Oddershede, J. Comp. Chem. 20, 1262 (1999).

[62]T. Enevoldsen, T. Rasmussen and S.P.A. Sauer, J. Chem. Phys. 114 (1), 84 (2001).

[63] T. Saue, Adv. Quant. Chem. 48, 383 (2005).

64]T. Saue, ChemPhysChem 12 (17), 3077 (2011).

65] J. Thyssen, T. Fleig and H.J.Aa. Jensen, J. Chem. Phys. 129 (3), 034109 (2008).

[66]H. Najib, N. Ben Sari-Zizi, J. Demaison, B. Bakri, J.M. Colmont and E.B. Mkadmi, J. Mol. Spectrosc. 220, 214 (2003).

[67]Y. Morino, K. Kuchitsu and T. Moritani, Inorg. Chem. 8, 867 (1969).

[68]H. Bürger, H. Ruland, J. Demaison and P. Dréan, J. Mol. Struct. 517, 105 (2000).

[69] J. Molnár, M. Kolonist and M. Hargittai, J. Mol. Struct. 413, 441 (1997). 
Table 1. Experimental structure parameters of the $\mathrm{XF}_{3}(\mathrm{X}=\mathrm{N}, \mathrm{P}, \mathrm{As}, \mathrm{Sb}, \mathrm{Bi})$ molecules.

\begin{tabular}{lccccc}
\hline & $\mathrm{NF}_{3}{ }^{\mathrm{a}}$ & $\mathrm{PF}_{3} \mathrm{~b}$ & $\mathrm{AsF}_{3}{ }^{\mathrm{c}}$ & $\mathrm{SbF}_{3} \mathrm{~d}$ & $\mathrm{BiF}_{3} \mathrm{~d}$ \\
\hline$R(\mathrm{XF}) / \mathrm{A}$ & 1.3676 & 1.5644 & 1.7043 & 1.880 & 1.987 \\
angle $(\mathrm{FXF}) /$ deg & 101.84 & 97.8 & 95.88 & 94.9 & 96.1 \\
\hline
\end{tabular}

${ }^{\mathrm{a}}$ Ref. 66, ${ }^{\mathrm{b}}$ Ref. 67] ${ }^{\mathrm{c}} \operatorname{Ref.68}{ }^{\mathrm{d}} \operatorname{Ref.69}$

Table 2. Magnetizabilities (in $e^{2} a_{0}^{2} / m_{e}$ ) of $\mathrm{XF}_{3}(\mathrm{X}=\mathrm{N}, \mathrm{P}, \mathrm{As}, \mathrm{Sb}, \mathrm{Bi})$. The relativistic effect $\Delta^{\text {rel }}$ is calculated as the difference between Dirac-Coulomb (DC) and non-relativistic (NR) results, the latter obtained with the Dalton code. The basis set notation pre-XZ refers to pre-cc-pVXZ basis sets of Dunning and co-workers 40 , 43 for the atoms F, N, P and As and corresponding basis sets by Dyall [44 45] for Sb and Bi.

\begin{tabular}{|c|c|c|c|c|c|c|c|c|c|c|}
\hline \multirow[b]{2}{*}{ Molecule } & & \multirow[b]{2}{*}{ Basis } & \multicolumn{4}{|c|}{ CGO } & \multicolumn{4}{|c|}{$\mathrm{LAO}$} \\
\hline & & & $\zeta_{\|}$ & $\zeta_{\perp}$ & $\zeta_{\text {iso }}$ & $\zeta_{\text {ani }}$ & $\zeta_{\|}$ & $\zeta_{\perp}$ & $\zeta_{\text {iso }}$ & $\zeta_{\text {ani }}$ \\
\hline \multirow{12}{*}{$\mathrm{NF}_{3}$} & \multirow[t]{9}{*}{$\mathrm{DC}$} & DZ & -14.83 & -9.78 & -11.47 & 5.04 & -4.27 & -4.87 & -4.67 & -0.60 \\
\hline & & $\mathrm{TZ}$ & -7.88 & -6.52 & -6.97 & 1.37 & -4.36 & -4.96 & -4.76 & -0.60 \\
\hline & & $\mathrm{QZ}$ & -5.65 & -5.54 & -5.58 & 0.11 & -4.43 & -5.04 & -4.83 & -0.61 \\
\hline & & aug-DZ & -6.75 & -6.31 & -6.46 & 0.44 & -4.57 & -5.22 & -5.00 & -0.65 \\
\hline & & aug-TZ & -5.01 & -5.42 & -5.28 & -0.41 & -4.64 & -5.24 & -5.04 & -0.60 \\
\hline & & aug-QZ & -4.70 & -5.26 & -5.08 & -0.56 & -4.64 & -5.24 & -5.04 & -0.60 \\
\hline & & d-aug-DZ & -6.37 & -6.18 & -6.24 & 0.19 & -4.57 & -5.22 & -5.00 & -0.65 \\
\hline & & d-aug-TZ & -5.01 & -5.45 & -5.31 & -0.44 & -4.69 & -5.27 & -5.08 & -0.58 \\
\hline & & d-aug-QZ & -4.76 & -5.31 & -5.13 & -0.55 & -4.68 & -5.28 & -5.08 & -0.60 \\
\hline & \multirow{3}{*}{$\begin{array}{c}\text { NR } \\
\text { Exp. }^{\text {a }} \\
\Delta^{\text {rel }}\end{array}$} & aug-QZ & & & & & -4.68 & -5.28 & -5.08 & -0.60 \\
\hline & & & & & & & & & & $-0.63( \pm 0.32)$ \\
\hline & & aug-QZ & & & & & 0.04 & 0.04 & 0.04 & 0.00 \\
\hline \multirow{10}{*}{$\mathrm{PF}_{3}$} & \multirow[t]{6}{*}{$\mathrm{DC}$} & $\mathrm{DZ}$ & -19.52 & -14.04 & -15.87 & 5.48 & -6.68 & -6.31 & -6.43 & 0.37 \\
\hline & & $\mathrm{TZ}$ & -10.39 & -9.18 & -9.59 & 1.21 & -6.66 & -6.27 & -6.40 & 0.39 \\
\hline & & $\mathrm{QZ}$ & -7.74 & -7.71 & -7.72 & 0.02 & -6.66 & -6.29 & -6.41 & 0.37 \\
\hline & & aug-DZ & -9.91 & -9.52 & -9.65 & 0.39 & -6.84 & -6.61 & -6.69 & 0.23 \\
\hline & & aug-TZ & -7.29 & -7.96 & -7.74 & -0.67 & -6.82 & -6.50 & -6.61 & 0.33 \\
\hline & & aug-QZ & -6.88 & -7.46 & -7.27 & -0.58 & -6.76 & -6.43 & -6.54 & 0.33 \\
\hline & \multirow{4}{*}{$\begin{array}{c}\text { NR } \\
\text { Exp. }^{\mathrm{a}} \\
\text { Exp. }^{\mathrm{b}} \\
\Delta^{\text {rel }}\end{array}$} & aug-QZ & & & & & -6.81 & -6.47 & -6.59 & 0.34 \\
\hline & & & & & & & & & $(-7.87)$ & $0.278( \pm 0.04)$ \\
\hline & & & & & & & & & & $0.286( \pm 0.04)$ \\
\hline & & aug-QZ & & & & & 0.06 & 0.04 & 0.05 & -0.01 \\
\hline \multirow[t]{8}{*}{$\mathrm{AsF}_{3}$} & \multirow[t]{6}{*}{$\mathrm{DC}$} & $\mathrm{DZ}$ & -23.87 & -19.08 & -20.68 & 4.80 & -8.30 & -8.01 & -8.11 & 0.29 \\
\hline & & $\mathrm{TZ}$ & -13.16 & -12.49 & -12.71 & 0.68 & -8.38 & -8.13 & -8.21 & 0.25 \\
\hline & & QZ & -9.85 & -10.39 & -10.21 & -0.55 & -8.39 & -8.17 & -8.24 & 0.22 \\
\hline & & aug-DZ & -12.15 & -12.26 & -12.22 & -0.10 & -8.48 & -8.31 & -8.37 & 0.17 \\
\hline & & aug-TZ & -9.12 & -10.29 & -9.90 & -1.18 & -8.50 & -8.30 & -8.37 & 0.20 \\
\hline & & aug-QZ & -8.62 & -9.77 & -9.39 & -1.14 & -8.49 & -8.29 & -8.36 & 0.20 \\
\hline & NR & aug-QZ & & & & & -8.59 & -8.36 & -8.44 & 0.23 \\
\hline & $\Delta^{\mathrm{rel}}$ & aug-QZ & & & & & 0.09 & 0.07 & 0.08 & -0.03 \\
\hline \multirow[t]{9}{*}{$\mathrm{SbF}_{3}$} & \multirow[t]{6}{*}{$\mathrm{DC}$} & $\mathrm{DZ}$ & -30.68 & -25.42 & -27.17 & 5.26 & -11.12 & -10.73 & -10.86 & 0.38 \\
\hline & & $\mathrm{TZ}$ & -17.28 & -17.18 & $\begin{array}{r}-17.22 \\
-17\end{array}$ & 0.10 & -11.22 & -10.91 & -11.01 & 0.30 \\
\hline & & QZ & -12.92 & -14.14 & -13.74 & -1.22 & -11.16 & -10.89 & -10.98 & 0.26 \\
\hline & & aug-DZ & -15.93 & -15.72 & -15.79 & 0.20 & -11.23 & -11.00 & -11.08 & 0.23 \\
\hline & & aug-TZ & -12.03 & -13.85 & -13.24 & -1.82 & -11.25 & -11.00 & -11.08 & 0.26 \\
\hline & & aug-QZ & -11.38 & -13.14 & -12.55 & -1.76 & -11.23 & -10.97 & -11.06 & 0.26 \\
\hline & \multirow{3}{*}{$\begin{array}{c}\text { NR } \\
\text { Exp. }^{\mathrm{c}} \\
\Delta^{\text {rel }}\end{array}$} & aug-QZ & & & & & -11.42 & -11.10 & -11.21 & 0.32 \\
\hline & & & & & & & & & -9.68 & \\
\hline & & aug-QZ & & & & & 0.19 & 0.13 & 0.15 & -0.06 \\
\hline \multirow{9}{*}{$\mathrm{BiF}_{3}$} & \multirow[t]{6}{*}{$\mathrm{DC}$} & $\mathrm{DZ}$ & -35.97 & -30.39 & -32.25 & 5.58 & -12.83 & -12.59 & -12.67 & 0.24 \\
\hline & & $\mathrm{TZ}$ & -20.44 & -19.08 & -19.53 & 1.36 & -12.80 & -12.68 & -12.72 & 0.13 \\
\hline & & QZ & -15.24 & -15.44 & -15.37 & -0.20 & -12.80 & -12.70 & -12.74 & 0.10 \\
\hline & & aug-DZ & -18.43 & -17.99 & -18.14 & 0.44 & -12.97 & -12.86 & -12.90 & 0.10 \\
\hline & & aug-TZ & -13.78 & -14.43 & -14.22 & -0.65 & -12.85 & -12.75 & -12.79 & 0.10 \\
\hline & & aug-QZ & -13.06 & -13.92 & -13.63 & -0.86 & -12.86 & -12.76 & -12.79 & 0.10 \\
\hline & NR & aug-QZ & & & & & -13.59 & -13.32 & -13.41 & 0.27 \\
\hline & Exp. ${ }^{d}$ & & & & & & & & -12.8 & \\
\hline & $\Delta^{\text {rel }}$ & aug-QZ & & & & & 0.73 & 0.56 & 0.62 & -0.17 \\
\hline
\end{tabular}

${ }^{\mathrm{a} R e f . ~ 54,}{ }^{\mathrm{b}}$ Ref. 55, ${ }^{\mathrm{c}}$ Ref. 49, ${ }^{\mathrm{d}}$ Ref. [57] 


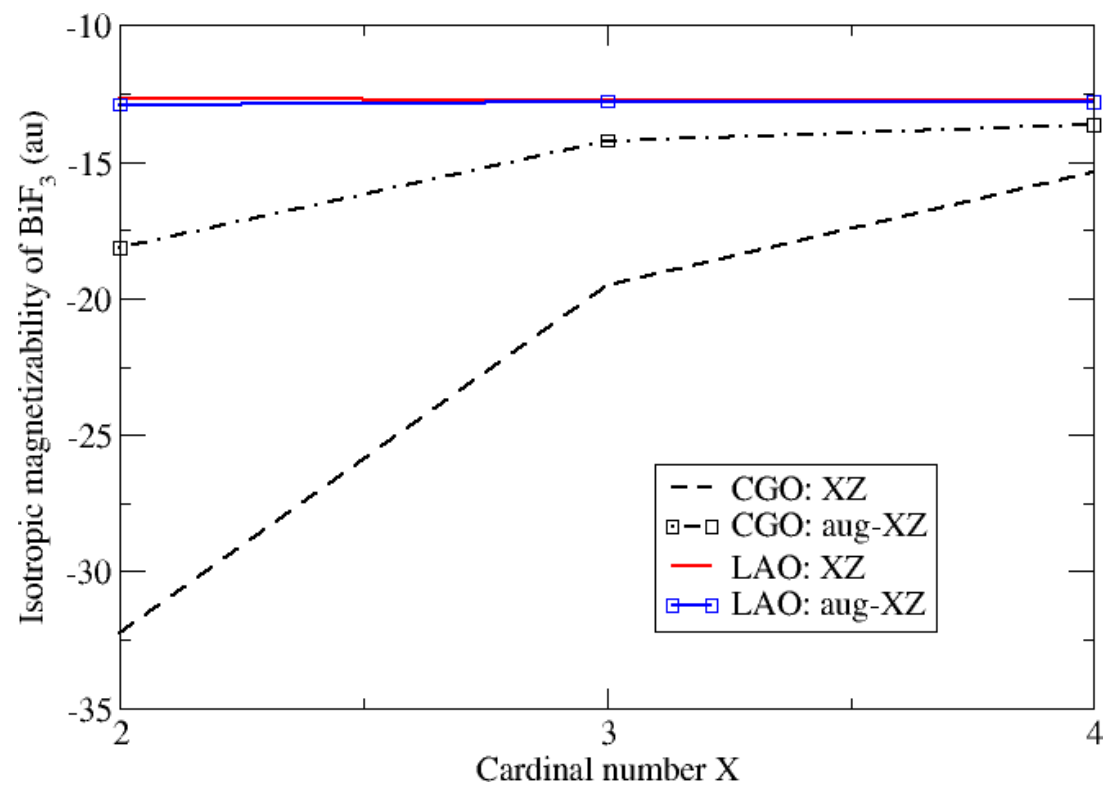

Figure 1. Convergence of $\zeta_{\text {iso }}$ (in $e^{2} a_{0}^{2} / m_{e}$ ) of $\mathrm{BiF}_{3}$ in $\mathrm{XZ}$ and aug-XZ basis sets using LAOs or CGO.
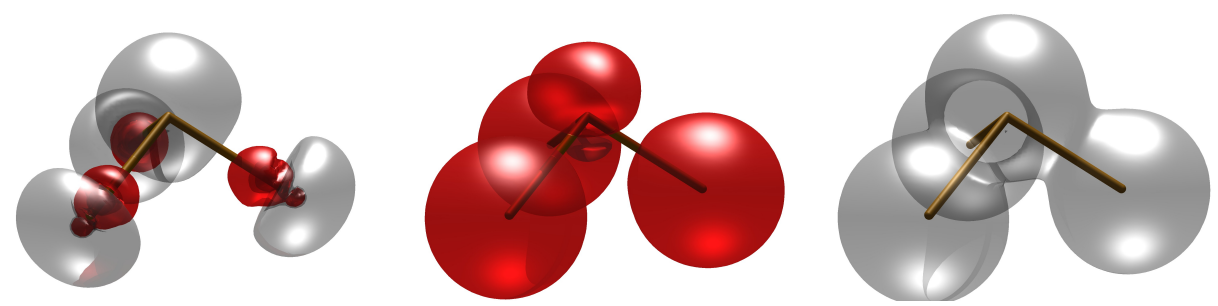

Figure 2. Isotropic average total (left), paramagnetic (middle), and diamagnetic (right) magnetizability density $\zeta_{\text {iso }}(\mathbf{r})$ in $\mathrm{BiF}_{3}$ (CGO aug-QZ; DC Hamiltonian; gray/light isosurface: $-0.035 e^{2} / m_{e} a_{0}$; red/dark isosurface: $\left.+0.035 e^{2} / m_{e} a_{0}\right)$.
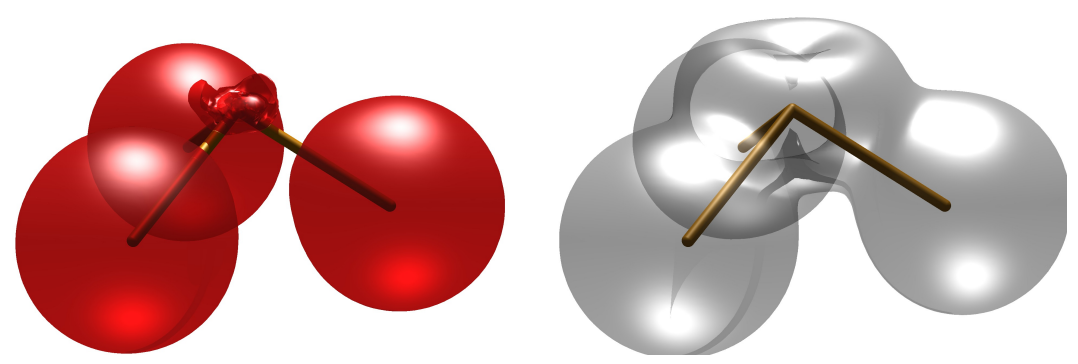

Figure 3. Parallel paramagnetic (left) and diamagnetic (right) magnetizability density $\zeta_{\|}(\mathbf{r})$ in $\mathrm{BiF}_{3}$ (CGO aug-QZ; DC Hamiltonian; gray/light isosurface: $-0.035 e^{2} / m_{e} a_{0}$; red/dark isosurface: +0.035 $\left.e^{2} / m_{e} a_{0}\right)$. 

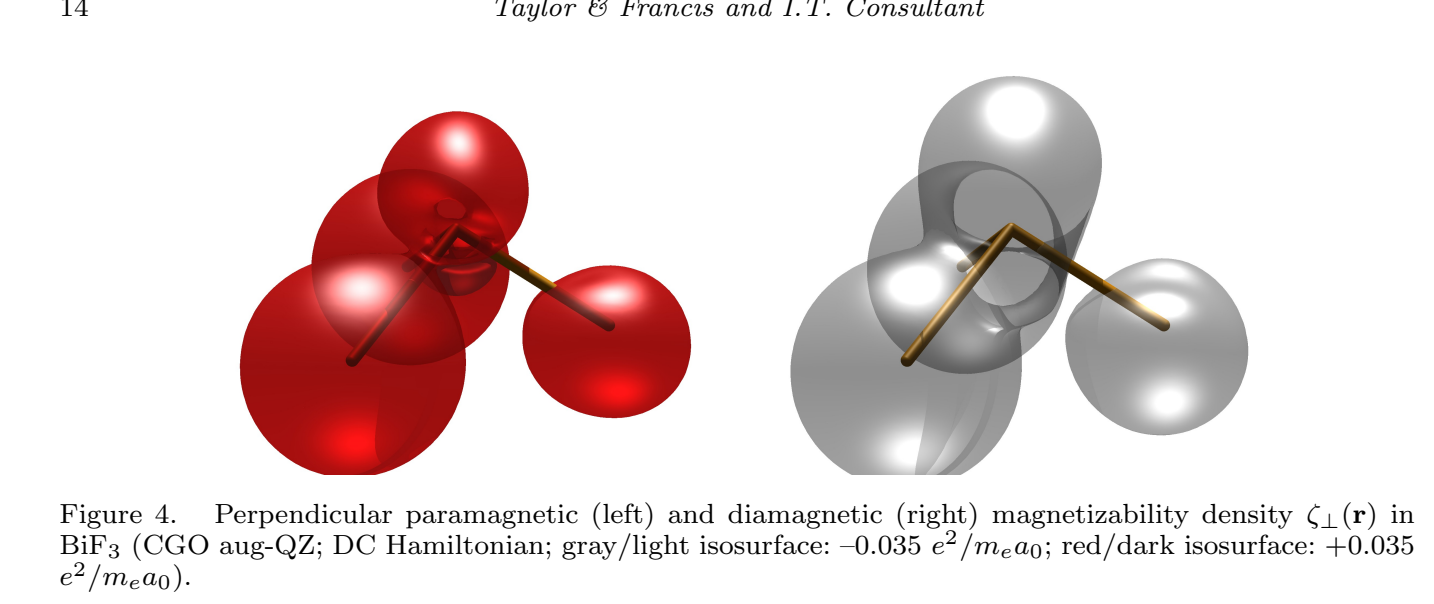

Figure 4. Perpendicular paramagnetic (left) and diamagnetic (right) magnetizability density $\zeta_{\perp}(\mathbf{r})$ in $\mathrm{BiF}_{3}$ (CGO aug-QZ; DC Hamiltonian; gray/light isosurface: $-0.035 e^{2} / m_{e} a_{0}$; red/dark isosurface: +0.035

14
Figure $4 ._{\text {BiF }}(\mathrm{CGO}$ au
$\left.e^{2} / m_{e} a_{0}\right)$.
(1)

Igure 4.
P
/ $\left.m_{e} a_{0}\right)$.

